Check for updates

Cite this: Chem. Sci., 2019, 10, 2945

๑ All publication charges for this article have been paid for by the Royal Society of Chemistry

Received 20th November 2018 Accepted 12th January 2019

DOI: $10.1039 / \mathrm{c} 8 \mathrm{sc} 05174 \mathrm{~g}$

rsc.li/chemical-science

\section{Light emission enhancement by supramolecular complexation of chemiluminescence probes designed for bioimaging $\dagger$}

\author{
Samer Gnaim, ${ }^{a}$ Anna Scomparin, (D) bc Anat Eldar-Boock, (D) ${ }^{\text {b }}$ Christoph R. Bauer, ${ }^{d}$ \\ Ronit Satchi-Fainaro (iD) ${ }^{\mathrm{b}}$ and Doron Shabat (iD) *a
}

\begin{abstract}
Chemiluminescence offers advantages over fluorescence for bioimaging, since an external light source is unnecessary with chemiluminescent agents. This report demonstrates the first encapsulation of chemiluminescence phenoxy-adamantyl-1,2-dioxetane probes with trimethyl $\beta$-cyclodextrin. Clear proof for the formation of a 1:1 host-guest complex between the adamantyl-1,2-dioxetane probe and trimethyl $\beta$-cyclodextrin was provided by mass spectroscopy and NMR experiments. The calculated association constant of this host-guest system, $253 \mathrm{M}^{-1}$, indicates the formation of a stable inclusion complex. The inclusion complex significantly amplified the light emission intensity relative to the noncomplexed probe under physiological conditions. Complexation of adamantyl-dioxetane with fluorogenic dye-tethered cyclodextrin resulted in light emission through energy transfer to a wavelength that corresponds to the fluorescent emission of the conjugated dye. Remarkably, the light emission intensity of this inclusion complex was approximately 1500 -fold higher than that of the non-complexed adamantyl-dioxetane guest. We present the first demonstration of microscopic cell images obtained using a chemiluminescence supramolecular dioxetane probe and demonstrate the utility of these supramolecular complexes by imaging of enzymatic activity and bio-analytes in vitro and in vivo. We anticipate that the described chemiluminescence supramolecular dioxetane probes will find use in various biological applications.
\end{abstract}




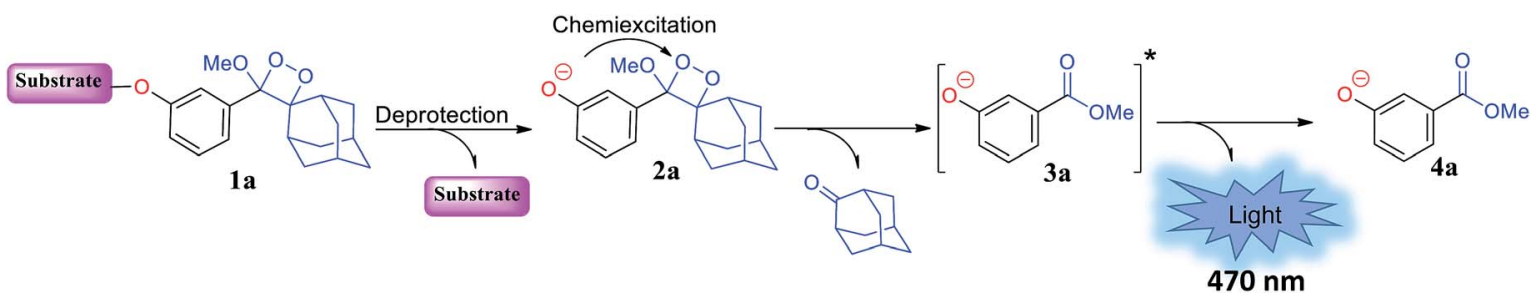

Fig. 1 Molecular structure and activation pathway of triggerable 1,2-dioxetane.

process known as chemically initiated electron exchange luminescence (CIEEL) to produce benzoate ester 3a in its electronically excited state. The decay of benzoate ester $\mathbf{3 a}$ to its ground state 4a is accompanied by emission of a blue photon. This class of 1,2-dioxetanes displays very high and efficient light emission in aprotic solvents such as dimethyl sulfoxide and acetonitrile. ${ }^{17}$

Under aqueous conditions, Schaap's dioxetanes suffer from major drawbacks of low solubility and low chemiluminescence quantum yield. ${ }^{20}$ The light emission efficiency is significantly decreased in aqueous solution relative to aprotic organic solvents as a result of non-radiative energy transfer that occurs upon interaction with surrounding water molecules.

This is likely due to the quenching effect of singlet excited anionic benzoate $\mathbf{3 a}$ caused by phenomena such as dipoledipole interactions, proton transfer, and hydrogen bonding between excited emitter $3 \mathbf{a}$ and water molecules. The low quantum yield of the chemiluminescence process in aqueous medium was improved to some extent by Schaap, Matsumoto, and others through the addition of a surfactant or by tethering a fluorescent dye to the 1,2-dioxetane molecule..$^{21-25}$

We have also explored new approaches to enhance the light emission of phenoxy-dioxetanes in water. A new methodology that significantly improved the emission ability of benzoate ester 3a under aqueous conditions, simply by installing an acceptor substituent at the ortho position of the phenoxy 1,2dioxetane, was recently reported. ${ }^{26}$ This structural motif afforded remarkable emission efficiency with significant chemiluminescence enhancement under physiological conditions. Our novel 1,2-dioxetane derivatives have also been demonstrated to be very efficient for in vitro and in vivo bioimaging..$^{27-32}$ In addition, our group has developed a modular practical synthetic route for dioxetane-fluorophore conjugates. We prepared two chemiluminescent dioxetane probes tethered to fluorescein or quinone-cyanine (QCy) dyes. ${ }^{33}$ The conjugates exhibited enhanced light emission efficiency under aqueous conditions compared to the parent probe.

The chemiluminescence light efficiency of Schaap's dioxetanes under aqueous conditions can also be improved through a supramolecular interaction with a host molecule that offers a hydrophobic protective environment. ${ }^{34}$ Host molecules such as cyclodextrins (CDs) can form an inclusion complex with the hydrophobic probe and stabilize the intermediate formed during the chemiexcitation reaction. Here, we report a new host-guest inclusion complex between triggerable phenoxy-1,2-dioxetane and trimethylated- $\beta$-cyclodextrin (TMCD) that is suitable for use under physiological conditions for in vitro and in vivo imaging.

\section{Results}

\section{Chemiluminescence enhancement effect obtained by complexation of adamantyl-dioxetane and CDs}

CDs are cyclic oligosaccharides formed by monomers of D-glucopyranose bound together by means of $\alpha-1,4$-glucosidic linkages that are closed into a ring to form a hollow truncated cone structure..$^{35}$ They are classified based on the number of linked monomers: $\alpha$-CDs consist of six units of glucose and $\beta$-CDs and $\gamma$-CDs consist of seven and eight glucose units, respectively. ${ }^{36}$ In a three-dimensional structure, the hydroxyl groups are on the outer edges, and the internal cavity contains only hydrogen atoms and oxygen bridges. Thus, CDs have an external hydrophilic surface and a hydrophobic central cavity. The central cavity of CDs (in particular that of $\beta$-CDs) can encapsulate hydrophobic molecules and, as a result, increase the solubility of the guest in water. ${ }^{37}$ CDs have been widely used in drug delivery, nucleic acid transfer, chiral separation of basic drugs, solubilization of lipophilic drugs, and molecular imaging..$^{38-44}$

It is well-known that adamantane guest molecules form highly stable complexes with $\beta$-CDs. ${ }^{40}$ The measured host-guest binding constant for adamantane with CDs is among the highest known for the formation of inclusion complexes because of the perfect fit of the adamantyl residue inside the $\beta$ CD cavity. ${ }^{45-47}$ As Schaap's dioxetane (I) contains an adamantyl moiety (Fig. 2A), ${ }^{48-50}$ we reasoned that CDs could be used to encapsulate 1,2-dioxetane probes. The $\beta$-CD-adamantane encapsulation process leads to formation of stable complex III. The CD cavity reduces water-induced quenching by providing a hydrophobic environment for the 1,2-dioxetane. Activation of the phenoxy 1,2-dioxetane generates excited state intermediate $\mathbf{I V}$, and its decay to the ground state should be accompanied by enhanced light emission (Fig. 2A). To further enhance and to modulate the color of the chemiluminescence light emission of dioxetane $\mathbf{I}$, the $\beta$-CD could covalently be attached to a fluorogenic dye as an energy transfer acceptor. In this way, the donor-acceptor space can be kept within the critical Foster distance by the host-guest complex. Energy transfer should take place efficiently from the donor (Schaap's dioxetane I) to $\beta$-CD-fluorogenic dye acceptor VII (Fig. 2B). Such energy transfer is expected to further enhance the light intensity under aqueous conditions with a red-shifted wavelength. ${ }^{\text {11-55 }}$

Alkaline-phosphatase (AP) probe 1 (Fig. 3A) was chosen to evaluate the CD-encapsulation effect on the chemiluminescence emission in water. Numerous $\alpha$-CD, $\beta$-CD, and $\gamma$ $\mathrm{CD}$ derivatives with various substituents on the hydroxyl groups 
A
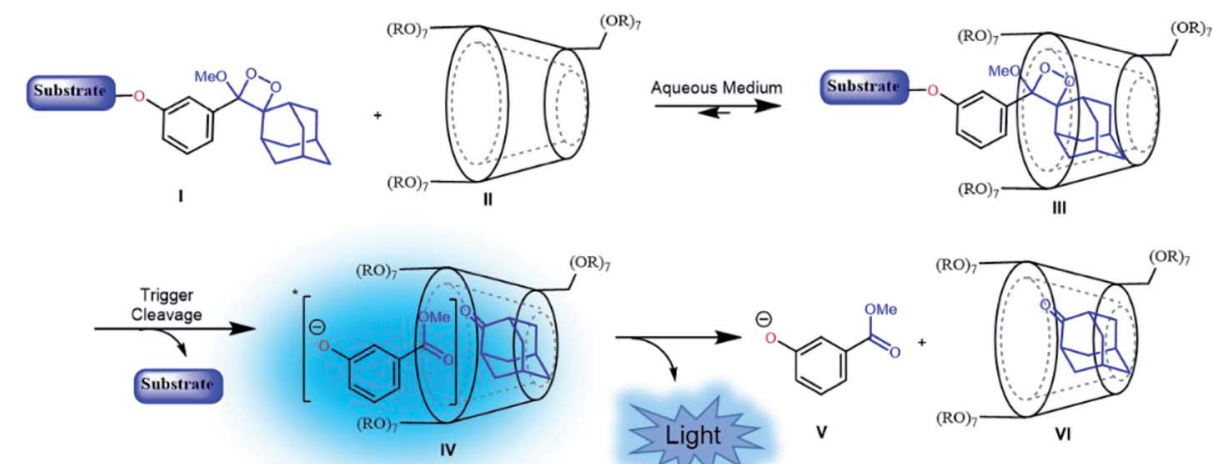

Light Emission Enhancement
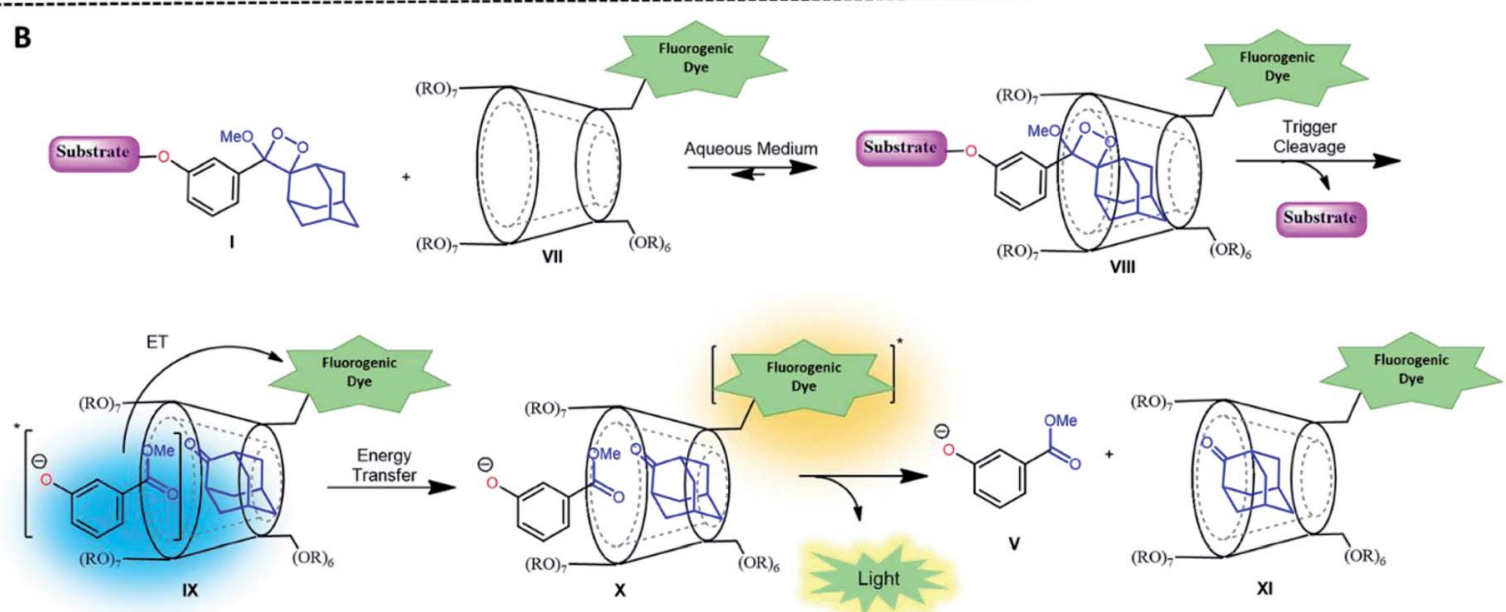

Fig. 2 (A) Encapsulation of triggerable dioxetane I with $\beta-C D$ derivatives. (B) Pathway of energy transfer of the probe in the presence of CDs tethered with a fluorophore.

A
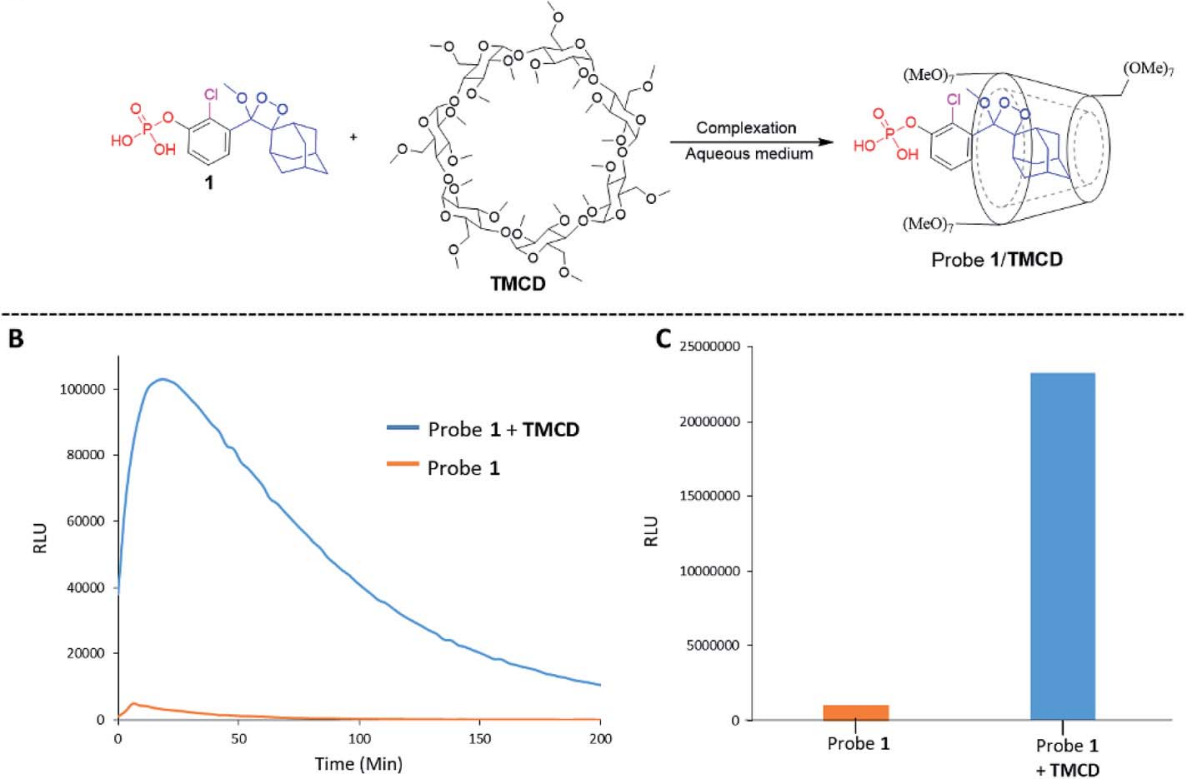

Fig. 3 (A) Chemical structures of probe 1 and TMCD. (B) Chemiluminescence kinetic profile and (C) total photon counts of probe 1 [0.5 mM] and TMCD $[9 \mathrm{mM}]$ in Tris buffer, $\mathrm{pH} 9$ (1\% DMSO) in the presence of 1 unit per $\mathrm{mL}$ alkaline phosphatase. 
were screened for their abilities to enhance the light emission of probe 1 in the presence of AP under aqueous conditions (Tris buffer, pH 9.0). The largest light emission enhancement effect was obtained with trimethylated- $\beta$-cyclodextrin (TMCD) as a host (Fig. 3A). This result could be explained through a structural circumstance, in which the secondary alcohols of the CD are located in close proximity to the adamantyl-dioxetane moiety encapsulation position. Such proximity could form hydrogen bond interactions with the dioxetane and its excited state intermediate.

These interactions lead to poor emission efficiency of Schaap's dioxetanes through a quenching effect. When the secondary alcohols are methylated, such a hydrogen bond quenching effect is avoided. The chemiluminescence light emission profile of probe $\mathbf{1}$ upon activation with AP was measured in the absence and presence of TMCD (Fig. 3B). The kinetic profile in the presence of TMCD was typical of a chemiluminescent probe with an initial signal increase to a maximum, in this case within 30 minutes, followed by a slow decrease to zero. TMCD significantly enhanced chemiluminescence with total photon counts emitted by probe 1 in the presence of TMCD being about 60-fold higher than those emitted by probe 1 in the absence of TMCD (Fig. 3C).

\section{Structural characterization and determination of the association constant between phenoxy-adamantyl-1,2- dioxetane and the TMCD host}

The formation of a non-covalent inclusion complex between the TMCD host and alkaline phosphatase 1,2-dioxetane probe 1 in aqueous solution was validated by electrospray ionization mass spectrometry (ESI-MS). ${ }^{56,57}$ Fig. $4 \mathrm{~A}$ shows the negative ion ESI spectrum acquired from an aqueous solution of TMCD and probe 1 in a molar ratio of $1: 1$. Three species are observed with $\mathrm{m} / \mathrm{z}$ values of $414.96,1463.23$, and 1845.18 , which adequately fit to $[\text { probe } 1]^{-},\left[\mathbf{T M C D}+\mathrm{Cl}^{-}\right.$, and the $1: 1$ complex $[\mathbf{T M C D}+$ probe 1$]^{-}$, respectively. This result indicates that there is a strong gas-phase interaction between the adamantyl moiety of probe 1 and the internal cavity of the CD derivative.

To provide additional evidence for the supramolecular structure obtained by the adamantyl probe and TMCD, we measured the ${ }^{1} \mathrm{H}$-NMR spectrum of supramolecular crystals of

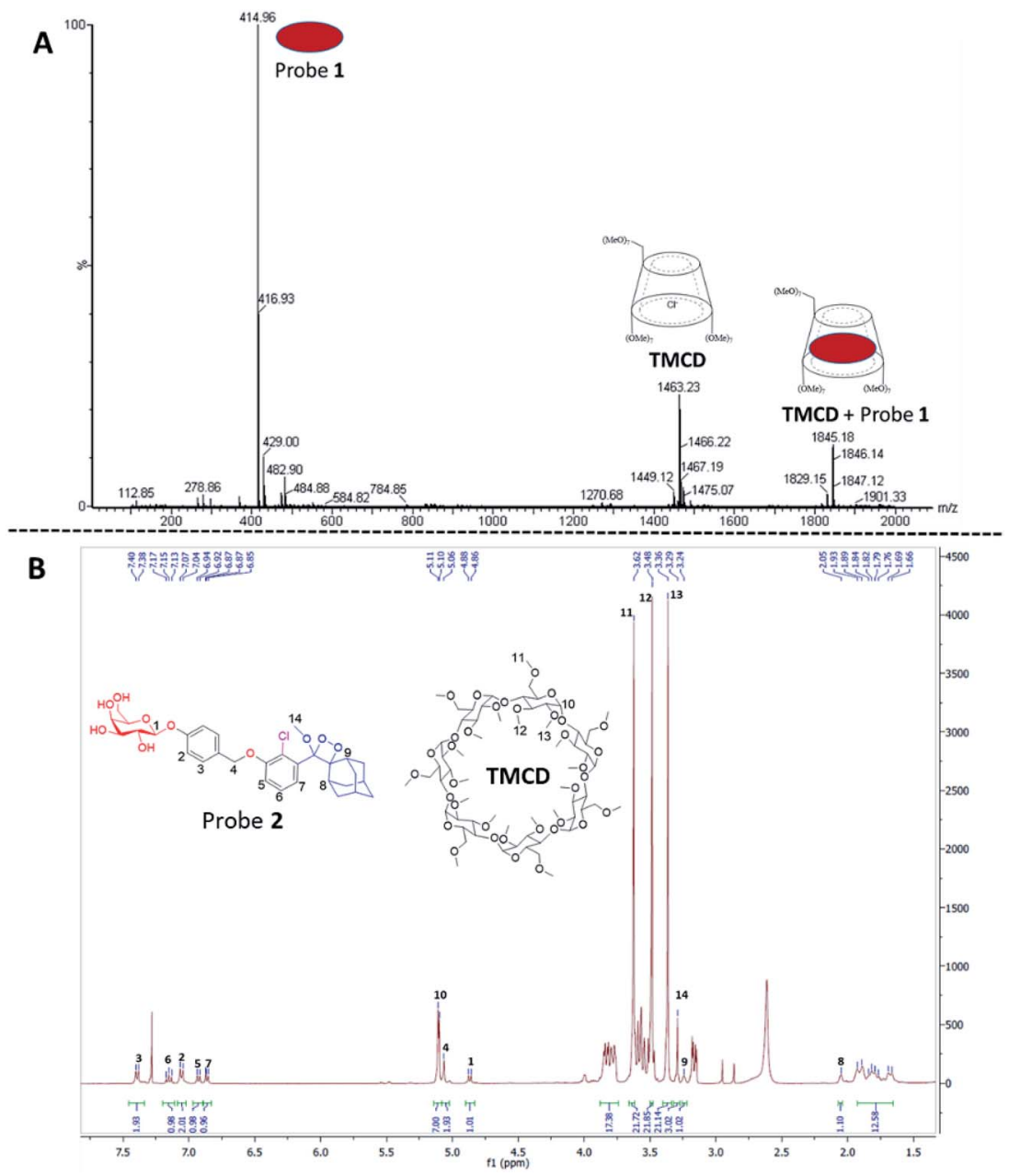

Fig. 4 (A) Negative ESI spectra of an aqueous solution of probe 1 and TMCD prepared in a $1: 1$ molar ratio. (B) ${ }^{1} \mathrm{H}-\mathrm{NMR}$ spectrum of the crystalline supramolecular complex of probe 2 with TMCD. The crystallization experiment was carried out in $\mathrm{H}_{2} \mathrm{O}: \mathrm{DMF}(95: 5)$ with probe 2 and TMCD mixed at an initial molar ratio of $1: 5$. 

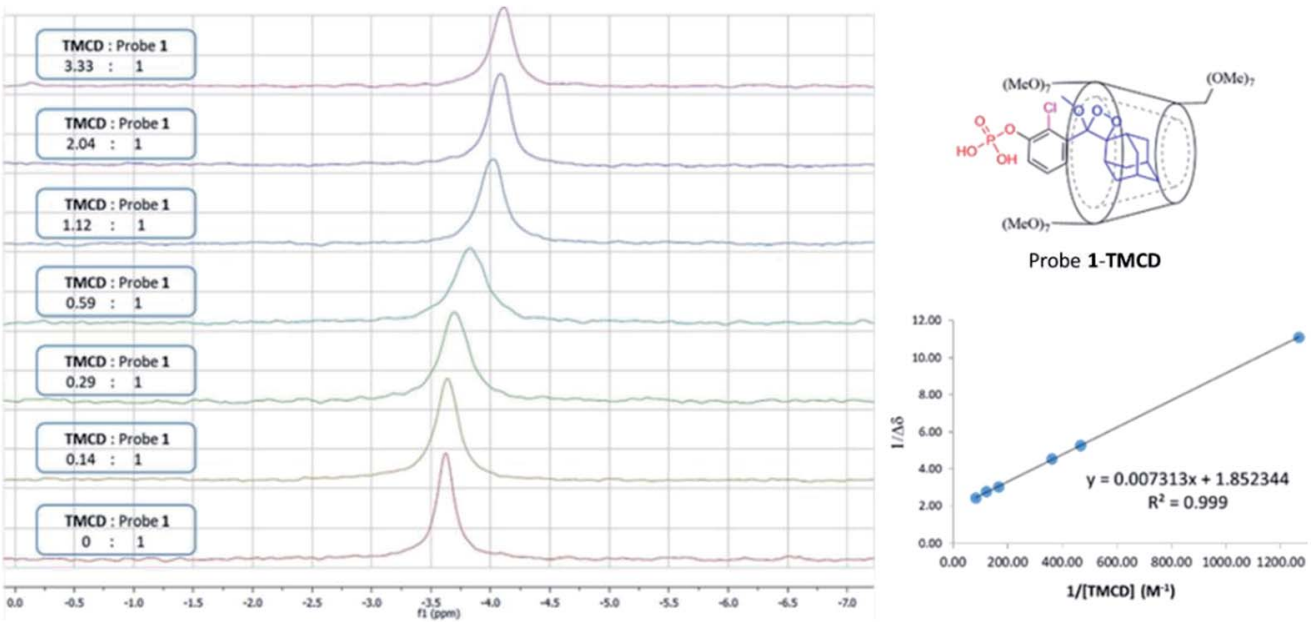

Fig. 5 (Left) ${ }^{31}$ P-NMR titration experiment of TMCD and probe 1. Conditions: $500 \mu \mathrm{L}$ of $\mathrm{D}_{2} \mathrm{O}, 5 \mathrm{mg}$ of probe 1 and up to $50 \mathrm{mg}$ of TMCD. (Right) Correlation of $1 / \Delta \delta$ as a function of probe $1 /[T M C D]$, based on the Benesi-Hildebrand equation.

the complex of probe 2 with TMCD obtained after mixing the two components at a ratio of $1: 5$ in aqueous medium. Probe 2 is a phenoxy 1,2-dioxetane equipped with a $\beta$-galactosidase substrate. The NMR signals clearly correspond to a $1: 1$ complex of probe 2-TMCD (Fig. 4B).

Next, ${ }^{31} \mathrm{P}$-NMR spectra were measured for solutions containing a fixed concentration of probe $1\left(5 \mathrm{mM}\right.$ in $\left.\mathrm{D}_{2} \mathrm{O}\right)$ and a variable concentration of the TMCD host (0.1-12.5 mM). Due to the chemical equilibrium of the complex formation, higher concentration of TMCD in the solution results in an increase in inclusion complex concentration. Consequently, the change of the ${ }^{31} \mathrm{P}$ chemical shift provides a direct indication of the concentration of the complex formed between the guest and the host. The Benesi-Hildebrand equation was used for determination of the binding constant based on the following parameters: $\Delta \delta$, the chemical shift change of the phosphor of probe $\mathbf{1}$;

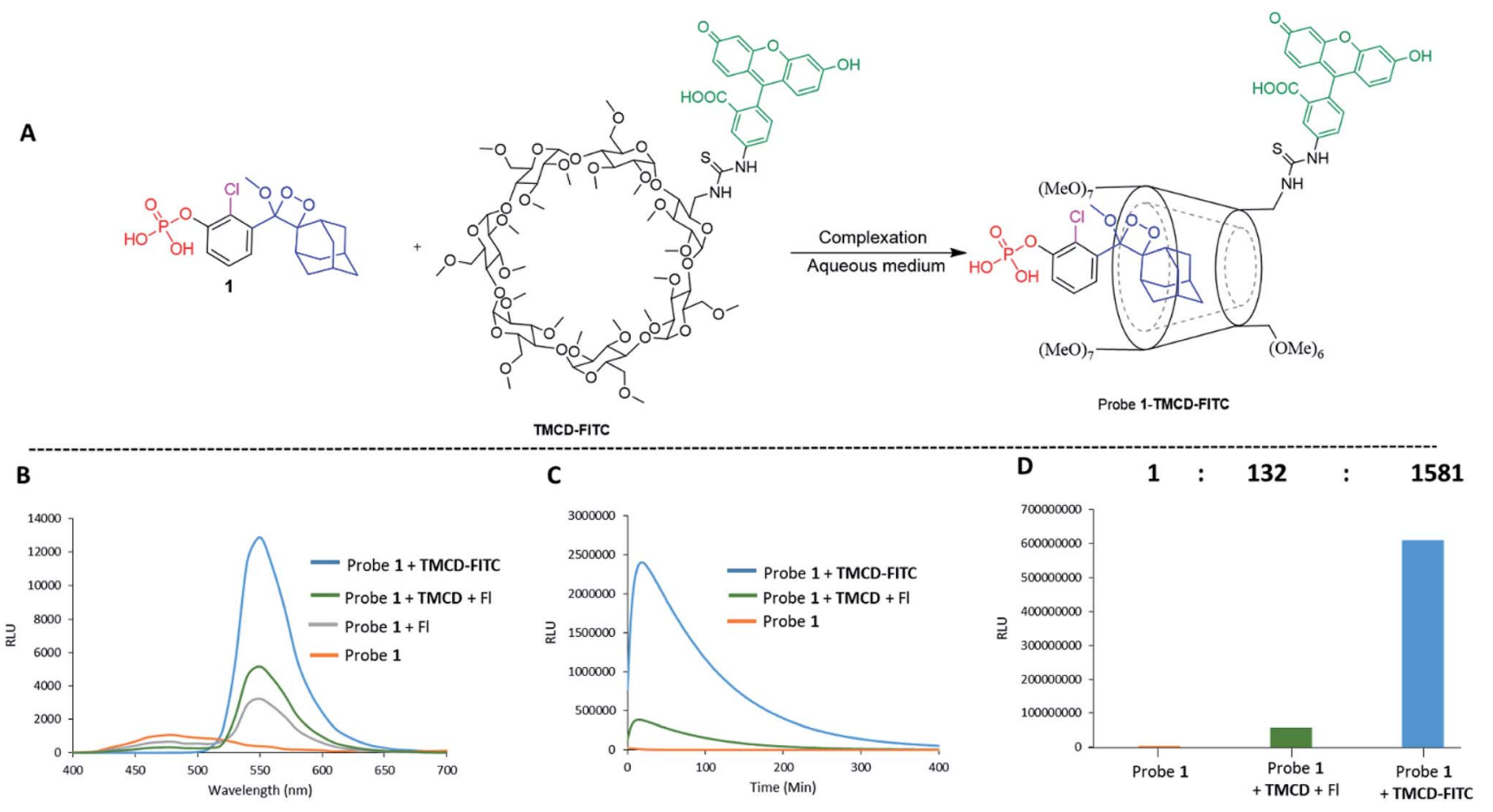

Fig. 6 (A) Chemical structures of probe 1 and TMCD-FITC. (B) Chemiluminescence spectra of probe 1 [0.5 mM], probe 1 [0.5 mM] + TMCD-FITC [5 mM], probe $1[0.5 \mathrm{mM}]+\mathrm{Fl}$ (fluorescein) $[5 \mathrm{mM}]$ and probe 1 [0.5 mM] + Fl [5 mM] + TMCD [5 mM] in Tris, pH 9 (1\% DMSO), in the presence of 1 units per $\mathrm{mL}$ alkaline phosphatase. (C) Chemiluminescence kinetic profile and (D) total photon count of probe 1 [0.5 mM], probe 1 [0.5 mM] + TMCD-FITC $[5 \mathrm{mM}]$ and probe $1[0.5 \mathrm{mM}]+\mathrm{TMCD}[5 \mathrm{mM}]+\mathrm{Fl}[5 \mathrm{mM}]$ in Tris, $\mathrm{pH} 9(1 \% \mathrm{DMSO})$, in the presence of $1 \mathrm{units}$ per $\mathrm{mL}$ alkaline phosphatase. 
$\Delta \delta_{\max }$, the maximum chemical shift difference upon full complexation; [TMCD], the concentration of the host; and $K$, the equilibrium constant of the complexation.

$$
\frac{1}{\Delta \delta}=\frac{1}{\left(K \Delta \delta_{\max }[\mathrm{TMCD}]\right)}+\frac{1}{\left(\Delta \delta_{\max }\right)}
$$

The ${ }^{31} \mathrm{P}-\mathrm{NMR}$ spectra of probe $\mathbf{1}$ upon incubation with various concentrations of TMCD are shown in Fig. 5. An excellent linear least-squares correlation was obtained by plotting $1 /$ $\Delta \delta$ as a function of $1 /[$ TMCD]. The plot clearly confirms a $1: 1$ intracavity complexation. The association constant $K$, calculated by determining the slope of the plot $\left[1 /\left(K^{*} \Delta \delta_{\max }\right)\right]$ and the intercept with the vertical axis $1 / \Delta \delta_{\max }$, was $253 \mathrm{M}^{-1}$. Comparison to previously reported examples ${ }^{58,59}$ clearly indicates that a relatively stable complex is formed between the phenoxyadamantyl-1,2-dioxetane probe and the TMCD host (about $70 \%$ of the probe was complexed under the applied experimental conditions when using the indicated maximum concentrations of the probe and TMCD).

\section{In vitro and in vivo imaging applications of adamantyl dioxetane-cyclodextrin complexes}

Next, we wanted to evaluate if energy transfer in a complex of probe 1 with TMCD conjugated to a dye would further enhance the light emission intensity of the chemiluminescence reaction. Following the strategy presented in Fig. 2B, we synthesized a conjugate of a fluorescein dye with the TMCD host molecule (see ESI $\uparrow$ for synthetic procedures). The chemical structure of the conjugate TMCD-FITC is shown in Fig. 6A. First, we measured the emission spectrum of probe $\mathbf{1}$ in comparison to that of a mixture of probe $\mathbf{1}$ and fluorescein, a mixture of probe 1, TMCD and fluorescein (Fl) and a mixture of probe $\mathbf{1}$ and TMCD-FITC upon activation by AP. The spectra of the probe 1 and fluorescein mixture and probe 1, TMCD and fluorescein mixture showed two emission maxima at wavelengths of 470 and $535 \mathrm{~nm}$, corresponding to the direct chemiluminescence of probe 1 and to the emission of fluorescein resulting from partial energy transfer. The spectrum of the probe 1 and TMCD-FITC mixture exhibited a single maximum emission wavelength of $535 \mathrm{~nm}$ (Fig. 6B). This observation clearly indicates that energy transfer from the chemiluminescent probe to the dye occurs and thus validates the significance of the supramolecular effect obtained between the dioxetane probe and the dye facilitated by the TMCD host.

To examine the chemiluminescence enhancement of probe 1 complexed with TMCD-FITC, comparative experiments were carried out in the presence and absence of the TMCD-FITC conjugate. In both of the cases probe 1 exhibited a typical chemiluminescent kinetic profile with the signal increasing to a maximum, followed by a slow decrease to zero. In the presence of TMCD-FITC, probe 1 exhibited a remarkably strong chemiluminescence emission signal under aqueous conditions; however, probe $\mathbf{1}$ in the absence of the host produced an extremely weak emission signal (Fig. 6C). The total light emitted by the probe 1-TMCD-FITC complex upon activation by AP was approximately 1500 -fold higher than that emitted by the probe without the host (Fig. 6D). Incubation of free TMCD, fluorescein dye and probe 1 under similar conditions led to minor light emission enhancement.

The activation of Schaap's chemiluminescence probes is based on removal of a protecting group from the phenolic

Table 1 Molecular structure and relative chemiluminescence parameters of probes 1-4 in the presence or absence of TMCD or TMCD-FITC

$\begin{array}{llll} & & \text { Relative CL emission } & \begin{array}{l}\text { Relative CL emission } \\ \text { Chemiluminescence probe }\end{array} \\ \text { (TMCD-FITC) } & \text { Enzyme/analyte } & \text { Buffer } & \text { (TMCD) }\end{array}$

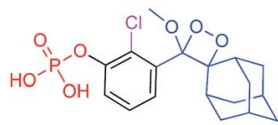

1
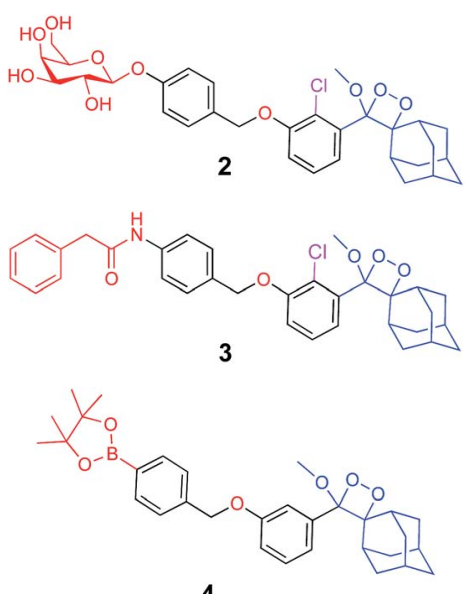

4
Alkaline phosphatase

Tris $\mathrm{pH}=9$

60

1581

$\beta$-Galactosidase

PBS $\mathrm{pH}=7.4$

15

PBS $\mathrm{pH}=8.3$

20
Hydrogen peroxide
Tris $\mathrm{pH}=10$

47
520

410

1444 
moiety. Therefore, different phenol protecting groups could be incorporated as triggering substrates for various analytes or enzymes. ${ }^{\mathbf{6 0 , 6 1}}$ To demonstrate the modularity of our supramolecular enhancement approach, we synthesized three additional probes for detection of the enzymes $\beta$-galactosidase and penicillin-G-amidase (PGA) and the analyte hydrogen peroxide (see ESI $\dagger$ for synthetic procedures). The molecular structures of probes and their relative chemiluminescence light emissions are presented in Table 1 . Probe 2 was equipped with a $\beta$-galactose group as a substrate for $\beta$-galactosidase, probe 3 with a phenylacetamide group as a substrate for PGA, and probe 4 with a phenylboronic ester as a substrate for hydrogen peroxide. Probes $\mathbf{2}$ and $\mathbf{3}$ were prepared with a chlorine substituent at the ortho position of the phenolic oxygen. The chlorine substituent was introduced in order to decrease the $\mathrm{pKa}$ of the released phenol obtained after the trigger removal. Such a pKa enables the chemiexcitation pathway of the dioxetane to occur under physiological conditions.

In comparative experiments, the chemiluminescence signals of the probes were measured in the presence and in the absence of TMCD or TMCD-FITC under the optimal pH conditions for the enzyme/analyte and the probes. The chemiluminescence emission of probe 2 upon activation with $\beta$-galactosidase in PBS, pH 7.4 was extremely weak. However, in the presence of TMCD-FITC, probe 2 exhibited a remarkably strong chemiluminescence emission signal with a 400-fold enhancement relative to the probe alone (15-fold for TMCD). In the presence of TMCD-FITC, probe 3 showed a similar signal enhancement, which is about 500-fold higher than that observed for probe 3 without the host upon the activation with the PGA enzyme in

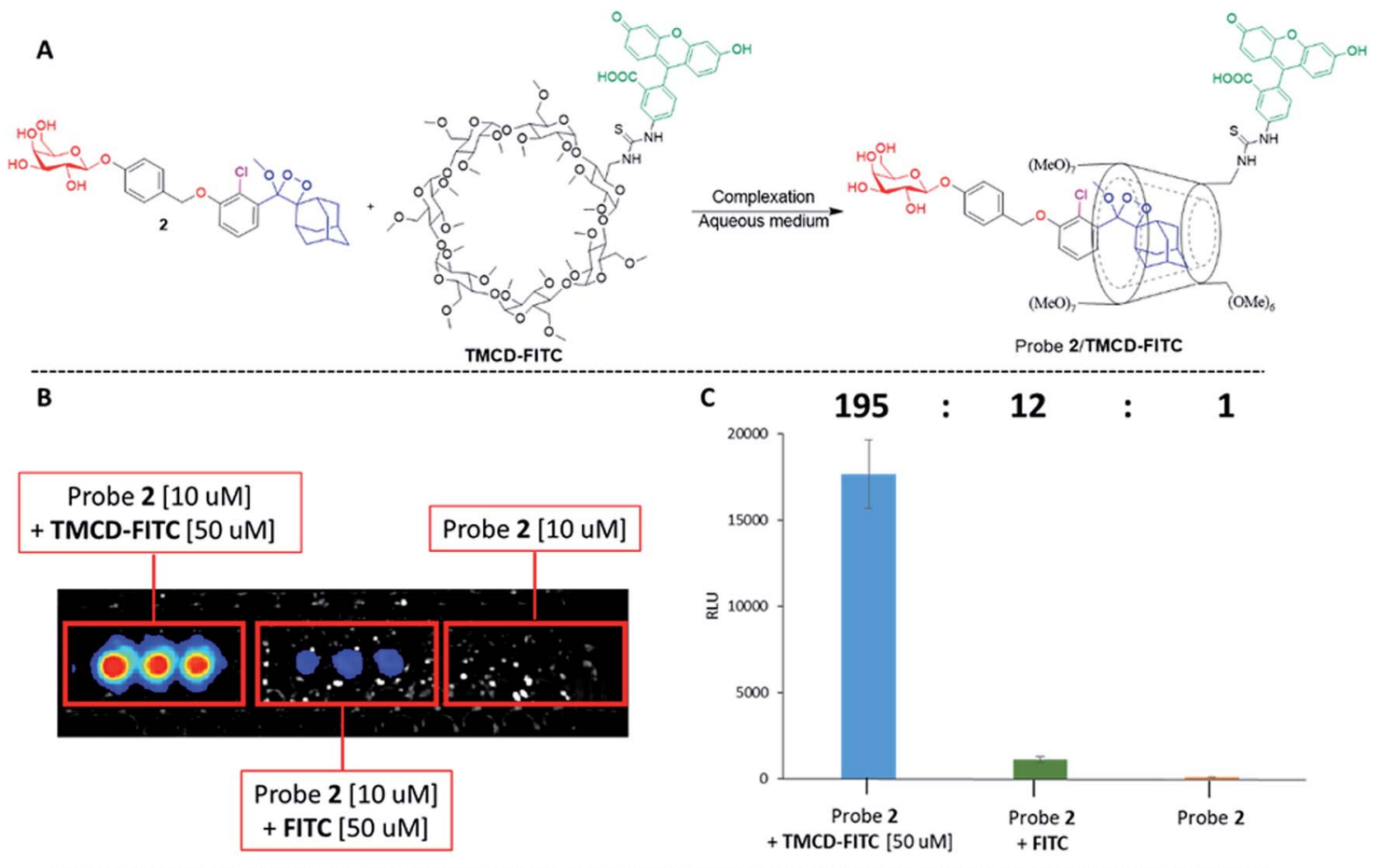

D

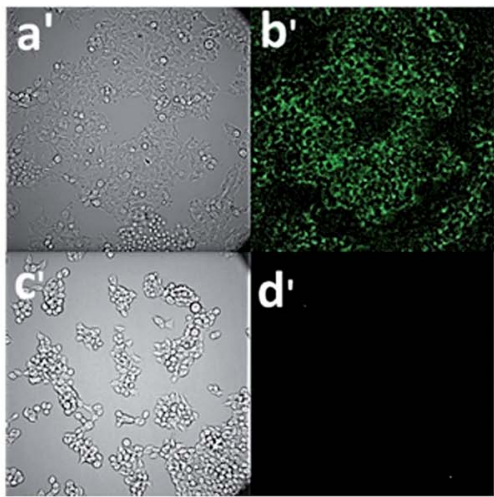

Fig. 7 (A) Chemical structures of probe 2 and TMCD-FITC. (B) Chemiluminescence imaging and (C) quantification of signal intensities in HEK-293-LacZ cells incubated with the complex of probe $2[10 \mu M]$ and TMCD-FITC [50 $\mu M]$. (D) (a') Transmitted light image and (b') chemiluminescence microscopy image of HEK-293-LacZ cells. $\left(c^{\prime}\right)$ Transmitted light image and ( $d^{\prime}$ ) chemiluminescence microscopy image of HEK293-WT cells. Images were obtained following 20 min of incubation with a cell culture medium containing probe 2 [50 $\mu$ M] and TMCD [50 $\mu$ M]. Images were taken using an LV200 Olympus microscope using a $60 \times$ objective and a 5 min exposure time. 
PBS, pH 8.3 (20-fold for TMCD). In the presence of TMCD-FITC, probe 4 exhibited the highest emission enhancement, almost 1500-fold higher than that of the probe without the host upon activation with hydrogen peroxide in Tris buffer, pH 10 (47-fold for TMCD).

The remarkable chemiluminescence enhancement obtained by complexation of TMCD-FITC with the phenoxy 1,2-dioxetane probes prompted us to examine whether the supramolecular complex could be used for live cell imaging. We first investigated the feasibility of the probe 2-TMCD-FITC complex for imaging endogenous $\beta$-galactosidase activity in human embryonic kidney cells (HEK293) transfected with the Lac-Z gene. Probe 2-TMCD-FITC, a mixture of probe 2 and FITC, and probe 2 were incubated with HEK-293-LacZ cells, and light emission was monitored (see ESI $\dagger$ for spectroscopic data). Remarkably, the probe 2-TMCD-FITC complex generated an intense chemiluminescence signal when incubated with HEK-293-LacZ cells, whereas negligible signals were observed with the probe 2 and FITC mixture and with probe 2 alone. The quantified chemiluminescence signals are presented in Fig. 7B. The ratio between the signal intensity observed for the probe 2-TMCDFITC complex and that of probe 2 alone was about 195-fold. These results clearly demonstrate that the TMCD-FITC-phenoxy 1,2-dioxetane system serves as a chemiluminescence probe that can be used to image $\beta$-galactosidase activity in living cells.

We next evaluated the ability of our probe system to image HEK-293-LacZ cells using an LV200 microscope. HEK-293-LacZ and HEK293 cells that do not express $\beta$-galactosidase (HEK-293WT) were incubated with the probe 2-TMCD-FITC complex and then imaged using an LV200 microscope (Fig. 7D). The obtained results clearly show that the supramolecular complex formed by probe $\mathbf{2}$ and TMCD-FITC was able to produce chemiluminescence images of the HEK-293-LacZ cells (Fig. 7D(b'). No chemiluminescence signal was observed from HEK-293-WT cells (Fig. 7D $\left(\mathrm{d}^{\prime}\right)$ ). Although the recorded image quality is rather low, this is the first successful attempt to obtain chemiluminescence images using a supramolecular complex of phenoxy 1,2dioxetanes.

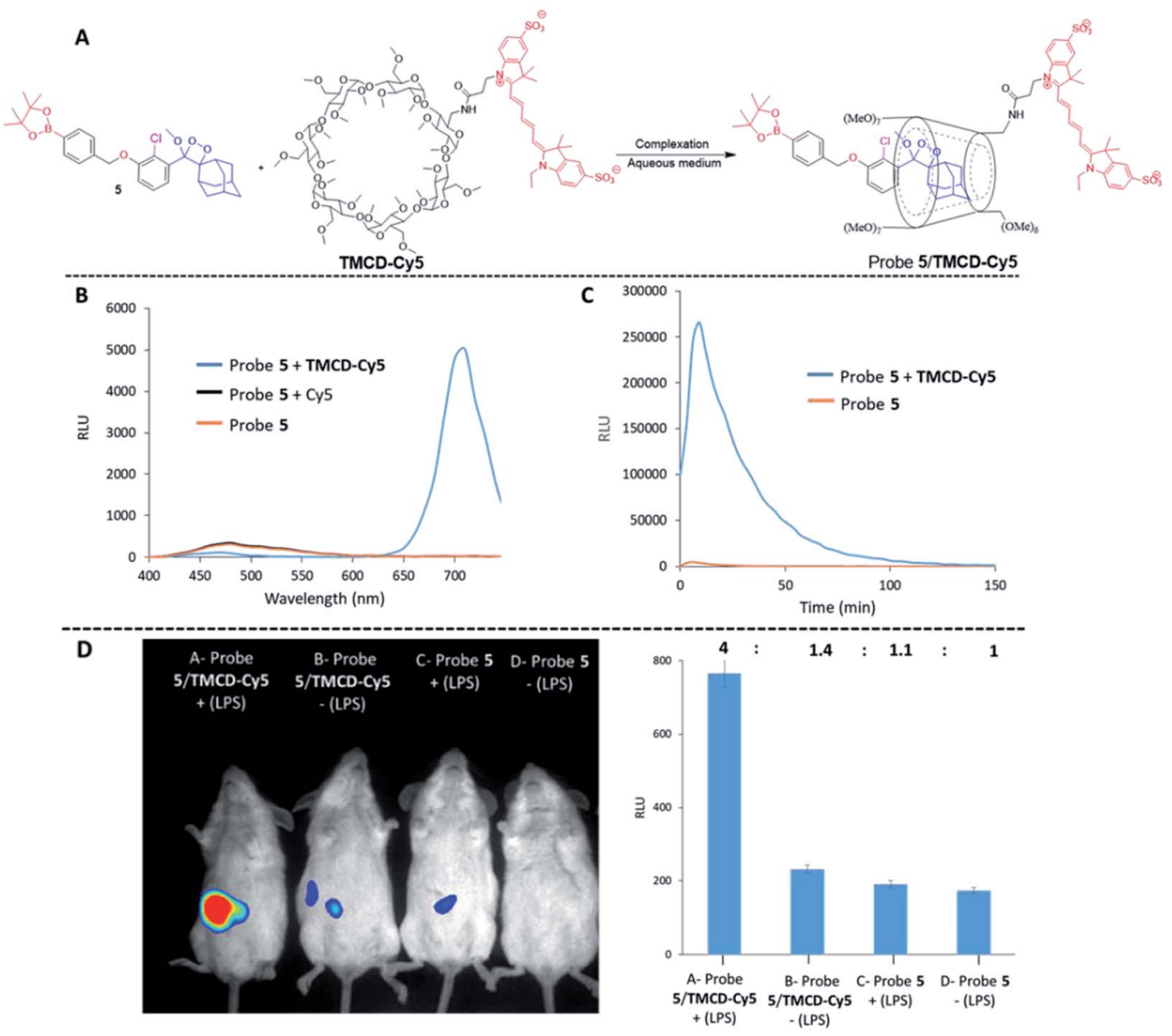

Fig. 8 (A) Chemical structures of probe 5 and TMCD-Cy5. (B) Chemiluminescence spectra and (C) kinetic profiles of probe 5 [100 $\mu$ M] and TMCD-Cy5 [300 $\mu \mathrm{M}]$ in PBS, pH 7.4 (1\% DMSO). (D) (Left) representative in vivo images of endogenous hydrogen peroxide in the peritoneal cavity of mice during an LPS-induced inflammatory response using probe 5 and TMCD-Cy5. The images of mice were recorded on a BioSpace Lab PhotonIMAGER. For the mice in group A, $1 \mathrm{~mL}$ of $0.1 \mathrm{mg} \mathrm{mL}^{-1}$ LPS was injected into the peritoneal cavity, followed $4 \mathrm{~h}$ later by an i.p. injection of probe $5[100 \mu \mathrm{M}]$ and TMCD-Cy5 [300 $\mu \mathrm{M}]$ in $100 \mu \mathrm{L}$ PBS, pH 7.4. For group B, $1 \mathrm{~mL}$ of PBS, pH 7.4 was injected i.p., followed $4 \mathrm{~h}$ later by an i.p.

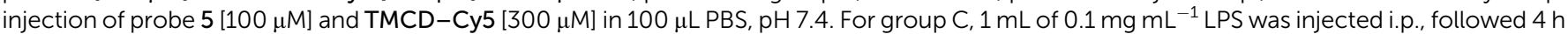
later by an i.p. injection of probe 5 [100 $\mu$ M] in $100 \mu \mathrm{L}$ PBS, pH 7.4. For group D, $1 \mathrm{~mL}$ of PBS, pH 7.4 was injected i.p., followed $4 \mathrm{~h}$ later by an i.p. injection of probe $5[100 \mu \mathrm{M}]$ in $100 \mu \mathrm{L}$ PBS, pH 7.4. (Right) quantification of signal intensities measured from each group of mice. 
Finally, we evaluated the use of the supramolecular phenoxydioxetanes as in vivo chemiluminescence probes. It was essential to synthesize a host system that was equipped with a nearinfrared (NIR) fluorescent dye for the in vivo application. The NIR region is useful for in vivo imaging, since such wavelengths better penetrate and are less scattered by living tissues than UV wavelengths. Using the strategy described in Fig. 2B, we synthesized the TMCD-Cy5 host conjugate by tethering TMCD with the Cy5 dye (see ESI $\dagger$ for synthetic procedures). Probe 5 can be used for imaging of reactive oxygen species (ROS) as it is equipped with a boronate trigger that can be activated with hydrogen peroxide. The chemical structure of probe 5 and the TMCD-Cy5 conjugate and the chemiluminescence enhancement properties upon reaction with hydrogen peroxide are shown in Fig. 8A.

No energy transfer was observed when a mixture of probe 5 was activated by hydrogen peroxide in solution in the presence of Cy5. However, the activation of probe 5 in the presence of TMCDCy5 resulted in almost complete energy transfer with light emission at a wavelength of $703 \mathrm{~nm}$ (Fig. 8B). The kinetic profiles of probe $\mathbf{5}$ with and without TMCD-Cy5 were measured in PBS, pH 7.4 in the presence of hydrogen peroxide. As shown in Fig. 8C, the signal produced by probe $\mathbf{5}$ complexed with TMCD-Cy5 was about 100-fold higher than that of probe 5 alone.

To demonstrate the ability of the chemiluminescence probe 5-NIR TMCD-Cy5 guest-host complex to serve as an imaging tool for in vivo use, we sought to image the biologically relevant ROS hydrogen peroxide. ${ }^{62}$ Since the overproduction of hydrogen peroxide in vivo is associated with the development of numerous inflammatory diseases, we investigated the ability of probe 5-TMCD-Cy5 to image endogenously produced hydrogen peroxide in a mouse model. Acute inflammation was induced by lipopolysaccharide (LPS). Mice were injected intraperitoneally (i.p.) with LPS ( $1 \mathrm{~mL}$ of $0.1 \mathrm{mg} \mathrm{mL} \mathrm{m}^{-1}$ ), followed 4 hours later by an additional i.p. injection of $100 \mu \mathrm{L}$ of probe $5[100 \mu \mathrm{M}]$ and TMCD-Cy5 $[300 \mu \mathrm{M}]$. Imaging was performed using a noninvasive BioSpace Lab PhotonIMAGER system. Fig. 8D shows that a significantly greater intensity of the NIR light emission signal was observed from LPS-treated mice injected with probe 5-TMCD-Cy5 (group A) compared with non-LPS-treated mice injected with the complex (group B). The slight light emission signal observed in non-LPS-treated mice is attributed to basal levels of hydrogen peroxide produced by living animals. In mice treated with LPS and in control untreated mice, probe $\mathbf{5}$ alone resulted in a significantly lower and more non-selective light emission signal than did the complex (groups $\mathrm{C}$ and D). The signal-to-noise ratio of the NIR light emission intensity observed from probe 5-TMCD-Cy5 in mice treated with LPS was about 3-fold higher than that of the non-LPS-treated mice.

\section{Discussion}

Currently, chemiluminescence signal enhancement is gaining increased attention. As mentioned above, our group recently reported the discovery of a new molecular methodology to significantly improve the light-emission efficiency of 1,2-dioxetane probes simply by installing various acrylate substituents at the ortho position of the phenoxy 1,2-dioxetane. The chemiluminescence light emission of the acrylate-substituted probes was up to 3000 fold higher than that of the known original adamantylidene-dioxetanes. In the present study, supramolecular complexation of the original 1,2-dioxetanes with cyclodextrin led to an increase of up to 1600 fold in chemiluminescence efficiency. Such a supramolecular approach possesses three key advantages: (i) there is no need for structural modification of the original Schaap's 1,2-dioxetane probe, (ii) complexation with fluorogenic dye-tethered cyclodextrin allows easy color modulation and red-shifting of the emitted light, and (iii) improved water solubility and chemical stability of the probe.

In commercial chemiluminescent immunoassays, a surfactant and a fluorescent dye are usually added together with adamantylidene-dioxetane probes. The surfactant reduces water-induced quenching by providing a hydrophobic environment for the excited chemiluminescent probe. The surfactantfluorescent dye component can enhance the light emission of the dioxetane probe under aqueous conditions by 400 -fold. However, since the surfactant mode of action relies on formation of micelles, its functional concentration is relatively high (above the critical micelle concentration, CMC). As micellar structures are not maintained when animals are treated systemically, the surfactant adduct approach is not practical for in vivo and cell applications.

\section{Conclusions}

In summary, we have demonstrated the first molecular encapsulation of chemiluminescence phenoxy-adamantyl-1,2dioxetane probes with trimethyl $\beta$-cyclodextrin. MS and NMR spectra provided clear proof for the formation of a stable $1: 1$ host-guest complex. The measured association constant of this host-guest system $\left(253 \mathrm{M}^{-1}\right)$ indicates the formation of a stable inclusion complex between phenoxy-adamantyl-1,2-dioxetanes and the trimethyl $\beta$-cyclodextrin. This inclusion complex was able to amplify the light emission intensity by 60 -fold under physiological conditions. Complexation of the adamantyldioxetane with fluorogenic dye-tethered cyclodextrin resulted in light emission through energy transfer to a longer wavelength, which corresponds to the fluorescent emission of the conjugated dye. Remarkably, the light emission intensity of such inclusion complexes was up to 1500 -fold higher than that of the adamantyl-dioxetane guest alone.

Overall, these supramolecular complexes were shown to serve as efficient chemiluminescence probes for imaging of enzymatic activity and bio-analytes in vitro and in vivo. In addition, we demonstrated the first microscopy cell images obtained using chemiluminescence supramolecular dioxetane probes. We anticipate that the described chemiluminescence supramolecular dioxetane probes would find further use in various biological applications.

\section{Ethics statement}

All animal procedures were performed in compliance with Tel Aviv University, Sackler School of Medicine guidelines and 
protocols approved by the Institutional Animal Care and Use Committee.

\section{Conflicts of interest}

There are no conflicts to declare.

\section{Acknowledgements}

D. S. thanks the Israel Science Foundation (ISF), the Binational Science Foundation (BSF) and the German Israeli Foundation (GIF) for financial support. S. G. thanks the Israeli Ministry of Science and Technology and the Council for Higher Education for the generous scholarships. RS-F laboratory is supported by the European Research Council (ERC) under the European Union's Seventh Framework Programme/ERC Consolidator Grant Agreement no. [617445]- PolyDorm.

\section{Notes and references}

1 A. T. Aron, K. M. Ramos-Torres, J. A. Cotruvo and C. J. Chang, Acc. Chem. Res., 2015, 48, 2434-2442.

2 M. H. Lee, E.-J. Kim, H. Lee, H. M. Kim, M. J. Chang, S. Y. Park, K. S. Hong, J. S. Kim and J. L. Sessler, J. Am. Chem. Soc., 2016, 138, 16380-16387.

3 M. H. Lee, A. Sharma, M. J. Chang, J. Lee, S. Son, J. L. Sessler, C. Kang and J. S. Kim, Chem. Soc. Rev., 2018, 47, 28-52.

4 S. W. Chou, Y. H. Shau, P. C. Wu, Y. S. Yang, D. B. Shieh and C. C. Chen, J. Am. Chem. Soc., 2010, 132, 13270-13278.

5 A. R. Kherlopian, T. Song, Q. Duan, M. A. Neimark, M. J. Po, J. K. Gohagan and A. F. Laine, BMC Syst. Biol., 2008, $2,74$. 6 M. Baker, Nature, 2010, 463, 977.

7 A. P. Dhawan, B. D'Alessandro and X. Fu, IEEE Rev. Biomed. Eng., 2010, 3, 69-92.

8 M. E. Quimbar, K. M. Krenek and A. R. Lippert, Methods, 2016, 109, 123-130.

9 S. Gross, S. T. Gammon, B. L. Moss, D. Rauch, J. Harding, J. W. Heinecke, L. Ratner and D. Piwnica-Worms, Nat. Med., 2009, 15, 455.

10 K. A. Jones, W. B. Porterfield, C. M. Rathbun, D. C. McCutcheon, M. A. Paley and J. A. Prescher, J. Am. Chem. Soc., 2017, 139, 2351-2358.

11 C. M. Rathbun and J. A. Prescher, Biochemistry, 2017, 56, 5178-5184.

12 L. Kricka, Anal. Chim. Acta, 2003, 500, 279-286.

13 C. Dodeigne, L. Thunus and R. Lejeune, Talanta, 2000, 51, 415-439.

14 S. Gnaim and D. Shabat, Chem. Commun., 2018, 54, 26552658.

15 S. Gnaim and D. Shabat, J. Am. Chem. Soc., 2017, 139, 1000210008.

16 S. M. Silva, K. Wagner, D. Weiss, R. Beckert, C. V. Stevani and W. J. Baader, Luminescence, 2002, 17, 362-369.

17 A. P. Schaap, T.-S. Chen, R. S. Handley, R. DeSilva and B. P. Giri, Tetrahedron Lett., 1987, 28, 1155-1158.

18 A. P. Schaap, R. S. Handley and B. P. Giri, Tetrahedron Lett., 1987, 28, 935-938.
19 A. P. Schaap, M. D. Sandison and R. S. Handley, Tetrahedron Lett., 1987, 28, 1159-1162.

20 M. Matsumoto, Y. Mizoguchi, T. Motoyama and N. Watanabe, Tetrahedron Lett., 2001, 42, 8869-8872.

21 M. Matsumoto, J. Photochem. Photobiol., 2004, 5, 27-53.

22 J. Y. Park, J. Gunpat, L. Liu, B. Edwards, A. Christie, X. J. Xie, L. J. Kricka and R. P. Mason, Luminescence, 2014, 29, 553558.

23 W. An, R. P. Mason and A. R. Lippert, Org. Biomol. Chem., 2018, 16, 4176-4182.

24 J. Cao, J. Campbell, L. Liu, R. P. Mason and A. R. Lippert, Anal. Chem., 2016, 88, 4995-5002.

25 J. Cao, R. Lopez, J. Thacker, J. Moon, C. Jiang, S. Morris, J. Bauer, P. Tao, R. Mason and A. Lippert, Chem. Sci., 2015, 6, 1979-1985.

26 O. Green, T. Eilon, N. Hananya, S. Gutkin, C. R. Bauer and D. Shabat, ACS Cent. Sci., 2017, 3, 349-358.

27 N. Hananya, O. Green, R. Blau, R. Satchi-Fainaro and D. Shabat, Angew. Chem., Int. Ed., 2017, 129, 11955-11958.

28 O. Green, S. Gnaim, R. Blau, A. Eldar-Boock, R. SatchiFainaro and D. Shabat, J. Am. Chem. Soc., 2017, 139, 13243-13248.

29 M. E. Roth-Konforti, C. R. Bauer and D. Shabat, Angew. Chem., Int. Ed., 2017, 56, 15633-15638.

30 S. Gnaim, O. Green and D. Shabat, Chem. Commun., 2018, 54, 2073-2085.

31 S. Gnaim, A. Scomparin, S. Das, R. SatchiFainaro and D. Shabat, Angew. Chem., Int. Ed., 2018, 57, 9033-9037.

32 J. Cao, W. An, A. G. Reeves and A. R. Lippert, Chem. Sci., 2018, 9, 2552-2558.

33 N. Hananya, A. Eldar Boock, C. R. Bauer, R. Satchi-Fainaro and D. Shabat, J. Am. Chem. Soc., 2016, 138, 13438-13446.

34 H. J. Sun, S. Zhang and V. Percec, Chem. Soc. Rev., 2015, 44, 3900-3923.

35 J. Szejtli, Chem. Rev., 1998, 98, 1743-1754.

36 M. E. Davis and M. E. Brewster, Nat. Rev. Drug Discovery, 2004, 3, 1023.

37 L. Szente and J. Szejtli, Adv. Drug Delivery Rev., 1999, 36, 1728.

38 (a) K. Uekama, F. Hirayama and T. Irie, Chem. Rev., 1998, 98, 2045-2076; (b) N. Mehwish, X. Dou, Y. Zhao and C. L. Feng, Mater. Horiz., 2019, 6, 14-44.

39 T. F. De Greef, M. M. Smulders, M. Wolffs, A. P. Schenning, R. P. Sijbesma and E. Meijer, Chem. Rev., 2009, 109, 56875754.

40 J. Michels, M. W. Baars, E. Meijer, J. Huskens and D. N. Reinhoudt, J. Biolumin. Chemilumin., 2000, 2, 19141918.

41 D. Zhang, Y. Nie, M. L. Saha, Z. He, L. Jiang, Z. Zhou and P. J. Stang, Inorg. Chem., 2015, 54, 11807-11812.

42 S. E. Sherman, Q. Xiao and V. Percec, Chem. Rev., 2017, 117, 6538-6631.

43 H. Wynberg, E. Meijer and J. Hummelen, Tetrahedron Lett., $1981,687$.

44 H. Goto, Y. Furusho and E. Yashima, J. Am. Chem. Soc., 2007, 129, 109-112. 
45 D. Granadero, J. Bordello, M. J. Pérez-Alvite, M. Novo and W. Al-Soufi, Int. J. Mol. Sci., 2010, 11, 173-188.

46 V. H. Tellini, A. Jover, L. Galantini, F. Meijide and J. V. Tato, Acta Crystallogr. B, 2004, 60, 204-210.

47 R. Maeztu, G. González-Gaitano and G. Tardajos, J. Chem., Biol. Phys. Sci., 2010, 114, 10541-10549.

48 R. Maeztu, G. González-Gaitano, G. Tardajos and P. Stilbs, J. Lumin., 2011, 131, 662-668.

49 M. Voicescu, G. Ionita, M. Vasilescu and A. Meghea, J. Inclusion Phenom. Macrocyclic Chem., 2006, 54, 217-219.

50 X. Ai, L. Niu, Y. Li, F. Yang and X. Su, Talanta, 2012, 99, 409414.

51 K. Teranishi and T. Nishiguchi, Anal. Biochem., 2004, 325, 185-195.

52 M. A. Hossain, H. Mihara and A. Ueno, J. Am. Chem. Soc., 2003, 125, 11178-11179.

53 G. Liang, J. W. Lam, W. Qin, J. Li, N. Xie and B. Z. Tang, Chem. Commun., 2014, 50, 1725-1727.

54 V. Percec, G. Johansson, G. Ungar and J. Zhou, J. Am. Chem. Soc., 1996, 118, 9855-9866.
55 Q. He, P. Tu and J. L. Sessler, Chem, 2017, 4, 46-93.

56 A. Jana, S. Bähring, M. Ishida, S. Goeb, D. Canevet, M. Sallé, J. O. Jeppesen and J. L. Sessler, Chem. Soc. Rev., 2018, 47, 5614-5645.

57 K. Sadrerafi, E. E. Moore and M. W. Lee, J. Inclusion Phenom. Macrocyclic Chem., 2015, 83, 159-166.

58 O. Swiech, K. Chmurski and R. Bilewicz, Supramol. Sci., 2010, 22, 461-466.

59 M. H. Lee, N. Park, C. Yi, J. H. Han, J. H. Hong, K. P. Kim, D. H. Kang, J. L. Sessler, C. Kang and J. S. Kim, J. Am. Chem. Soc., 2014, 136, 14136-14142.

60 L. Long, M. Huang, N. Wang, Y. Wu, K. Wang, A. Gong, Z. Zhang and J. L. Sessler, J. Am. Chem. Soc., 2018, 140, 1870-1875.

61 K. Li, J. T. Hou, J. Yang and X. Q. Yu, Chem. Commun., 2017, 53, 5539-5541.

62 X. Zhen, C. Zhang, C. Xie, Q. Miao, K. L. Lim and K. Pu, ACS Nano, 2016, 10, 6400-6409. 